SOFÍA GURIDI

WAAG SOCIETY

AMSTERDAM, HOLANDA

SGURIDI@UC.CL
Fecha de recepción: 28/06/2019

Fecha de aceptación: 05/10/2019

Cómo citar: Guridi Sotomayor, S. (2019)

Técnicas dialogantes. Arpillera y las nuevas

tecnologías de e-textiles

RChD: creación y pensamiento, 4 (7), 1-14

DOI: $10.5354 / 0719-837 X .2019 .53722$

\section{Técnicas dialogantes: arpillera y las nuevas tecnologías de e-textiles}

\author{
Convergence of Techniques: Traditional Textile Technique of \\ Arpillera and e-textiles Technologies
}

Resumen. La artesanía desde su identidad basada en el hacer manual, el arte y la cultura, no siempre se ha visto relacionada con el mundo de las tecnologías. Sin embargo, los avances del último tiempo en desarrollo de materiales inteligentes y nuevas plataformas tecnológicas de código abierto han permitido el surgimiento de un nuevo concepto "Computational Craft" que alude justamente a esta unión virtuosa entre computación y tradición artesanal. La siguiente publicación aborda cómo los avances en la creación de textiles electrónicos pueden acercarse al mundo de las técnicas tradicionales, potenciando particularmente las características expresivas y comunicativas de la técnica chilena de la arpillera. Para ello, se expondrá el trabajo de investigación aplicada desarrollado por la autora en el marco del curso internacional Fabricademy: A new textile and technology academy.

Palabras clave: arpilleras, artesanía, e-textiles, interacción, tecnología.

\begin{abstract}
From its identity based in hand making and art and culture, crafts have not always been related to the world of technologies. However, the advances made in recent times in the development of intelligent materials and new open-source platforms, have allowed the emergence of the new concept 'Computational Crafts' referring precisely to a virtuous union between computing and artisanal tradition. The following publication discusses how advances in the creation of electronic textiles can approach the world of traditional techniques, particularly enhancing the expressive and communicative characteristics specific to the Chilean embroidering art of Arpilleras.

As a case of study, the author presents the research project developed in the framework of the International course Fabricademy: A new textiles and technology academy, featuring the preliminary results.
\end{abstract}

Keywords: arpilleras, crafts, e-textiles, interaction, technology. 


\section{Introducción}

En los últimos años, e impulsado en gran medida por el desarrollo de Internet, la tecnología se ha acercado cada vez más a todas las esferas de nuestra vida. Componentes electrónicos cada vez más pequeños se agregan a objetos de la vida cotidiana, ya sean autos, instrumentos musicales, pasaportes o incluso nuestras mascotas (Persson, 2013). En el campo particular de los textiles, las tecnologías han tenido siempre un rol fundamental en el desarrollo de nuevos métodos de fabricación, como fue por ejemplo la introducción de la máquina Jacquard para el tejido de patrones, pero también en la creación de nuevos materiales cada vez más sofisticados, como sucede con los textiles para el rubro aeroespacial o el deporte de alto rendimiento.

Durante los últimos treinta años ha surgido una nueva rama dentro de la innovación textil, asociada más bien al mundo de la electrónica y el diseño de interacción llamada textiles electrónicos o e-textiles. Gracias al desarrollo de nuevos materiales y técnicas, como las fibras e hilos conductores, los chips y procesadores cada vez más pequeños, se ha abierto un mundo de exploración sobre cómo integrar sensores, actuadores y procesadores de información directamente en los textiles y el vestuario. Una de las ramas más relevantes que ha surgido a partir de esto son los wearables o "vestibles", los cuales apuntan a aumentar las capacidades humanas y de conexión a través de objetos vinculados al cuerpo, como la camisa FysioPal de Pauline van Dongen, capaz de detectar malas posturas en el usuario y enviar información a un smartphone (Guler, Gannon y Sicchio, 2016).

Estos proyectos, en general, toman en cuenta el carácter "utilitario" de las nuevas tecnologías de información susceptibles de integrarse en los textiles, especialmente los asociados con el cuerpo. Ahora bien, lo que nos interesa en este escrito es plantear la pregunta sobre cómo estos nuevos materiales y soportes pueden vincularse, no solo al desarrollo de productos con un fin utilitario y destinados a la industria productiva, sino también a técnicas textiles tradicionales asociadas al campo de la artesanía y la cultura. Si los nuevos materiales y técnicas son presentados como propulsores de la innovación y transformación (Berzowska, 2005), y para considerar el énfasis que se ha puesto en el trabajo entre artesano y diseñador para la continuidad del conocimiento tradicional (UNESCO, 2009), es que parece atinente trabajar en esta temática de colaboración. Bajo esta premisa, se toma el concepto de "Computational Craft" el cual alude a esta unión entre computación y tradición artesanal, en la que ambas partes aportan desde lo simbólico, material y técnico. Las experiencias de este tipo han sido desarrolladas por artistas y diseñadoras internacionales, como es el caso de Amor Muñoz con su trabajo "Yucatech" y el de Maggie Orth, investigadora de MIT Media Lab.

Para ello, la siguiente publicación aborda el caso de estudio "Arpilleras Parlantes", proyecto de investigación aplicada llevada a cabo por la diseñadora Sofía Guridi en el marco del curso internacional Fabricademy: A new textile and technology academy, realizado en Waag Society, Amsterdam durante 2017-2018 y finalizado en Santiago de Chile. Su objetivo principal es explorar desde una primera aproximación si las nuevas tecnologías de e-textiles, desde su potencial para crear interfaces de intercambio de información interactivas, pueden o no vincularse a una técnica tradicional textil con foco en la narración de historias, para aportar al desarrollo de nuevos productos que potencien su carácter comunicativo, identitario e interactivo. Para ello, se plantea la creación de una pieza textil interactiva, desarrollada junto con la 
agrupación "Arpilleristas de Melipilla", que sigue una metodología que incluye tanto investigación, prototipado y cocreación entre diseñadora y artesanas. La elección de la técnica de la arpillera para este proyecto tiene que ver con su valor como soporte cultural, sus medios expresivos (patchwork, sobredimensión de los personajes, colores saturados) y la intención principal de contar historias a través de la tela. Sumado a esto, se ve una oportunidad de acercar esta técnica a nuevo público, inicialmente internacional (dada la naturaleza del curso Fabricademy).

Por otro lado, se buscará conocer el punto de vista de artesanas, su valoración respecto al tema y las ideas que surjan desde sus propias inquietudes. Junto a esto, se espera ahondar en los conocimientos de e-textiles, donde se utilice información open-source para la adaptación de la electrónica a la técnica particular del bordado. Finalmente, se espera dar a conocer los resultados y generar discusión respecto a la temática con entes de diversos medios, ya sean artesanos, académicos, diseñadores o público general.

\section{Marco teórico}

Son tres las nociones que subyacen en esta exposición: e-textiles, computational craft y arpillera en tanto arte textil. A continuación, precisamos cada uno de esos términos.

\section{E-textiles}

Desde su origen, el textil como soporte ha estado vinculado a la tecnología, ya que es clave para el desarrollo de nueva maquinaria, de procesos productivos y materiales. Tanto los telares industriales como los textiles con fibras de cobre antibacteriales de la empresa chilena The Copper Company son ejemplos de este vínculo virtuoso que ha traído consigo innovaciones para el mundo del vestuario, el mobiliario, y las áreas altamente sofisticadas a nivel técnico como el desarrollo aeroespacial, la medicina o la alta performance deportiva. Particularmente, durante los últimos cincuenta años, la industria textil ha vivido los cambios más revolucionarios en cuanto se refiere a innovación. La unión entre ciencia de los materiales, mecánica estructural, inteligencia artificial y biología ha permitido el desarrollo de smart materials, los cuales pueden definirse como materiales y estructuras capaces de sensar, reaccionar y adaptarse a condiciones del ambiente o diversos estímulos, ya sean mecánicos, térmicos, eléctricos, magnéticos u otras fuentes (Tao, 2001). Este desarrollo, sumado a los avances en el mundo de la tecnología computacional y electrónica, es el que ha permitido entonces el surgimiento de los e-textiles o textiles electrónicos. De acuerdo a la definición dada por la investigadora Joanna Berzowska (2005), el concepto se refiere a "sustratos textiles que incorporan la capacidad de sensar, de comunicar (usualmente wireless), transmitir energía, y tecnologías de interconexión para permitir a sensores o dispositivos de procesamiento de información, estar conectados entre sí por medio del textil” (p. 4). Algunas de las áreas de investigación que han tenido mayor incidencia en este desarrollo son la creación de fibras textiles conductoras de electricidad, fibra óptica, polímeros conductores, tintas termocrómicas capaces de cambiar de color según la temperatura, micro y nanomateriales y miniaturización de componentes electrónicos. Los e-textiles entonces han permitido sumar la capacidad de captar, procesar y generar información del cuerpo y el ambiente al textil, lo que tiene un impacto positivo en el desarrollo de proyectos vinculados con monitoreo de signos vitales en medicina, análisis 


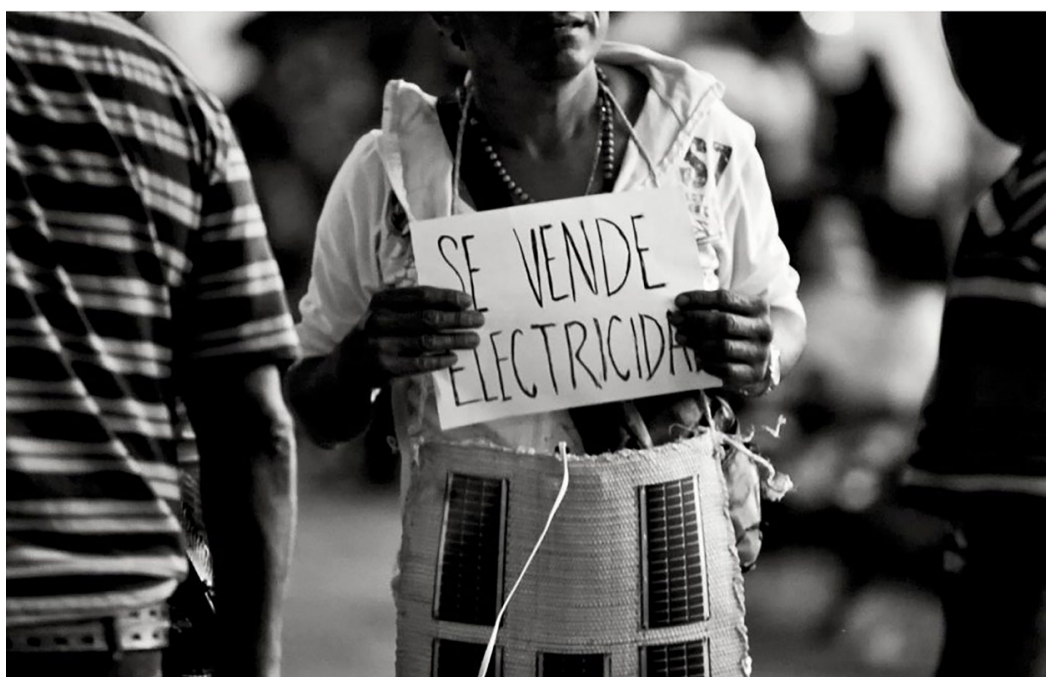

de datos para mejorar la performance de deportistas de alto rendimiento, seguridad y defensa militar, y creación de vestuario artístico.

\section{Computational craft}

Junto a los avances realizados por la industria de alta tecnología, ha surgido un nuevo género comúnmente llamado Computational crafts. Las crafts o artes manuales, tradicionalmente se asocian al uso de materiales y técnicas low-tech, pero poco a poco han surgido espacios de encuentro entre estas y el mundo de la computación, lo cual ha sido beneficioso para ambos.

Por un lado, la computación con toda su capacidad de procesamiento de información ha permitido realzar las capacidades expresivas de materiales y técnicas tradicionales. Desde el punto de vista de las artes, estas pueden acercar la computación al mundo táctil y físico, para crear nuevos soportes que también contribuyan a la transferencia de conocimientos de manera más concreta y lúdica. Según Blauvelt, Wrensch y Eisenberg (2000) es posible distinguir tres estrategias de unión. En primer lugar, el uso de software como una herramienta con la cual diseñar objetos que luego serán fabricados de manera artesanal. Así, el uso de códigos de programación que integren variables como geometría o cantidad de pliegues puede permitir el desarrollo de diseños complejos que luego puedan a su vez ser compartidos por redes colaborativas online, para abrir los rangos de posibilidades. Desde el mundo textil, un ejemplo de esto es el desarrollo de softwares para patronaje como Seamly2D. En segundo lugar, la creación de objetos de manera artesanal pero utilizando smart materials que permitan nuevos resultados; por ejemplo, el uso de tintas termocrómicas reactivas a la temperatura. Finalmente, la forma más ambiciosa de unión de estos dos mundos tiene que ver con generar sistemas donde sea posible usar una variedad de materiales tradicionales e "inteligentes", en la que alguno de ellos permita la comunicación entre objetos y todo esto sea acompañado de un software que permita programar algunos de los elementos.

Desde lo textil, el caso más interesante es el proyecto "LilyPad" de Leah Buckley, el cual utiliza como base de desarrollo el hardware y software de programación Arduino, para crear un kit de e-textiles que permite, a usuarios inexpertos, crear piezas textiles interactivas incorporando electrónica bordada. Este kit abrió el camino para que personas de todo el mundo tuvieran 
la oportunidad de explorar la unión de e-textiles y técnicas tradicionales, para compartir información online en plataformas como Kobakant, Adafruit o High Low Tech. Como ejemplo de esta rama del computational craft asociado al textil, tenemos el caso de "Yuca_tech" de la artista Amor Muñoz, la cual al integrar paneles solares sobre tejidos tradicionales mexicanos permite la producción de energía eléctrica desde un bolso o los zapatos.

\section{Arpillera como arte textil y soporte narrativo}

El arte textil elegido para el desarrollo de este proyecto de unión entre e-textiles y artesanía es la arpillera. Esta técnica basada en el bordado de cortes de tela sobre sacos de papa, tiene su origen durante la dictadura militar en Chile a mediados de los años 70 . Se inició pensada como un medio para generar recursos a través de la capacitación de mujeres bordadoras y se transformó en un medio de comunicación y denuncia pública de la violación de derechos humanos a ciudadanos chilenos. Las mujeres arpilleristas retratan a través del hilo y tela escenas de crímenes, personas desaparecidas o casas sin padres ni hijos. Así, la arpillera se instaló como un soporte para narrar historias y vivencias con valor cultural, identitario y político, para unir la transmisión del oficio del artesano con una mirada testimonial, aliada a la memoria colectiva.

El discurso de la arpillera no es especulativo ni teórico, es concreto y vivencial, centrado en una costura especifica que, por medio de códigos perfectamente descifrables, testimonia lo que la voz no puede exclamar, o como en un textum literario, las arpilleras cuentan una historia. Por medio de los rústicos hilos se preserva una memoria colectiva, escrita-cosida por aquellas anónimas y tantas veces ignoradas mujeres. (Agosin, 1985)

En términos formales, la creación de las arpilleras tiene como fuente de inspiración primera los tapices de Violeta Parra y bordados realizados por mujeres de pescadores de Isla Negra, hechos en lana sobre tela rústica. Sin embargo, durante el periodo de dictadura, las arpilleras aplicaron retazos de tela e incluso restos de ropa propia o de sus familiares, los cuales son bordados con puntadas básicas tipo festón, puntada atrás, puntada simple o "pata de gallo", realizadas con lana partida e hilo.

Si bien los contenidos de la narración pueden ser variados, ya sea desde la denuncia de crímenes o la representación de la vida cotidiana, la composición de las arpilleras se ha mantenido a través del tiempo. Esta se destaca principalmente por tener siempre presente el cielo, la cordillera de los Andes y la tierra como base para agregar las demás figuras que conformaran las escenas: "Con las diferentes texturas y colores, las arpilleristas crearon efectos de cercanía y lejanía, espacio, dimensiones o volumen, detalles que se pueden apreciar especialmente en la representación de la cordillera, una constante en las arpilleras y en las escenas de acción" (Museo de la Memoria y los Derechos Humanos, 2012).

Finalmente, un detalle característico es la utilización de materiales variados como alambre o fósforos y la incorporación de personajes tridimensionales, los cuales representan a distintas entidades de la sociedad chilena, como mujeres, carabineros, religiosos e incluso animales.

Actualmente, esta técnica sigue vigente y llevada a cabo por grupos de mu- 
Figura 2. Arpillera "Los estudiantes quemados" Carmen Gloria y Rodrigo. Fuente: archivo digital Museo de la Memoria.

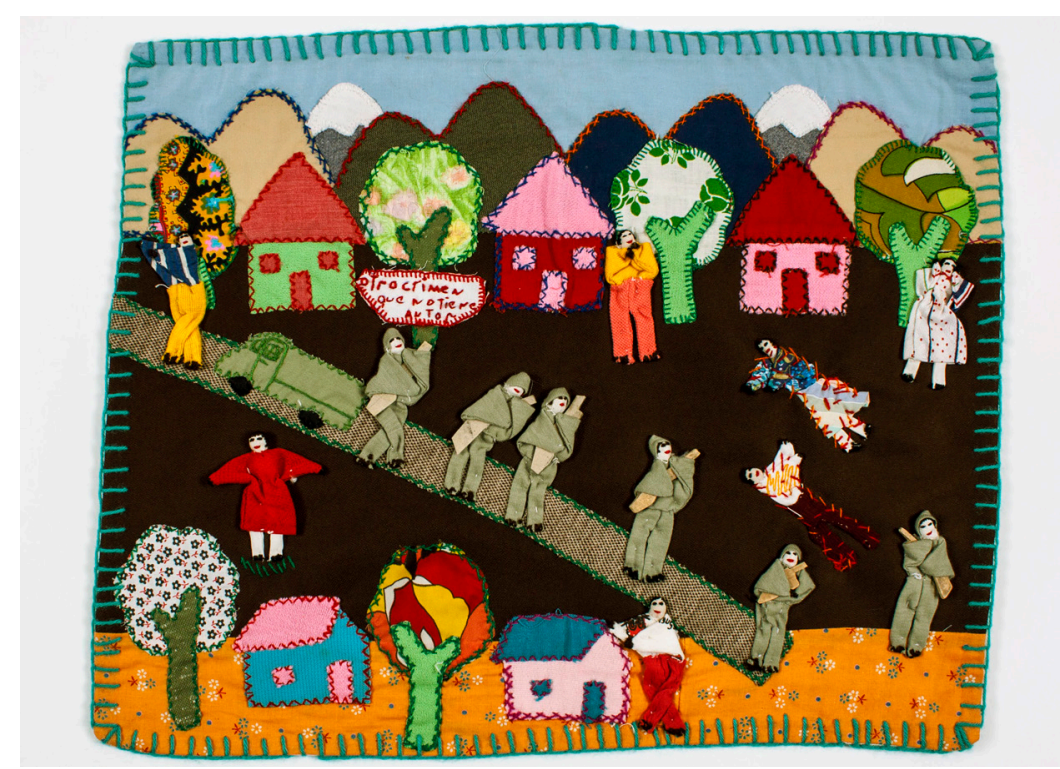

jeres, principalmente en la zona central de Chile. Parte de estos grupos como Arpilleristas de Melipilla, Arpilleristas de lo Hermida o el colectivo Memorarte: Arpilleras urbanas, continúan con la creación de piezas que representan tanto la contingencia social nacional como también retratos de la vida del campo y las ciudades, junto con sus mitos y costumbres como la hilandería de lana o la fabricación del pan.

Si se considera lo expuesto, la elección de la arpillera tiene relación con su gran capacidad narrativa gracias a su técnica expresiva y tridimensional, lo cual se alinea con las características de intercambio de información propias de los e-textiles. Su soporte textil accesible al tacto (a diferencia de una obra pictórica), presenta potencial para generar soportes interactivos que potencien el vínculo entre el observador y la obra. Al tomar en cuenta el carácter exploratorio del proyecto de vinculación entre la técnica tradicional y nuevas tecnologías de comunicación, esta técnica en particular es lo suficientemente flexible como para permitir esta adaptación de ambas partes. Si bien mantiene una composición base, las historias o "escenas" particulares varían de arpillera en arpillera, para dar espacio a la creación de nuevas historias que consideren por ejemplo, el uso del tacto, sonido, luz o movimiento. Por otro lado, con la intención de acercar la técnica a nuevos públicos y generar ingresos a las mujeres artesanas, esta técnica ha evolucionado durante los últimos años y ya no son solo tapices, sino también tarjetas de celebración, carteras e incluso libros como El copihue: narraciones populares en arpillería, por lo cual parece atingente aportar desde una nueva perspectiva como es la mirada del diseño con nuevas tecnologías como una forma de explorar la creación de nuevos productos, que respete siempre la esencia de la técnica y su valor como representación de la memoria colectiva.

\section{Metodología}

El desarrollo del proyecto fue guiado por una metodología de investigación, prototipado y co-creación, que tiene como meta la creación de una pieza textil interactiva en colaboración con la agrupación Arpilleristas de Melipilla. En primer lugar, fue necesario adentrarse en el mundo de los e-textiles y la articulación 
entre electrónica, programación, textil y diseño desde la perspectiva del "Hazlo tú mismo", donde se utilizan materiales especializados pero disponibles en el mercado y programación en Arduino. Desde el inicio de los e-textiles, y a la par de la industria tecnológica de alta gama, han surgido iniciativas que buscan explorar y transmitir conocimientos sobre ellos desde una mirada más craft/ Hazlo tú mismo. Un ejemplo de esto es el proyecto "Kobakant" llevado a cabo por Mika Satomi y Hannah Perner-Wilson, el cual entrega información a la comunidad de makers interesados, a través de una plataforma online nutrida por tutoriales y ejemplos de proyectos. Estos siguen criterios como el bajo costo de materiales, el uso de herramientas textiles tradicionales como máquinas de coser y bordado tradicional para llegar, por ejemplo, a fabricar guantes con sensores de presión que reaccionan al movimiento de los dedos del usuario. El primer periodo de trabajo, entonces, tuvo que ver con el estudio de electrónica básica, programación, uso de microcontroladores y sensores, y creación de material e-textiles a partir de plataformas de código abierto como la ya mencionada, u otras como Adafruit.com, Highlowtech.com y Arduino.cc. Una vez terminada la etapa de estudio e investigación, se continuó con el prototipado y creación de muestras. El foco fue crear sensores y actuadores utilizando componentes ya disponibles en el mercado como fotorresistencias o tela conductora, junto con las técnicas estudiadas sobre soldado de partes, bordado y creación de placas electrónicas flexibles de bajo costo. Las principales muestras desarrolladas fueron sensores de presión, de tacto, botones, sensores de luz, diodos electroluminiscentes, motores de vibración, reproductores de sonido y luces RGB. Estos componentes fueron vinculados a través de diversos microcontroladores como Lilypads, Gemma y Attiny85.

Finalmente, y con un extenso desarrollo de muestras que permitiera entender las posibilidades de creación de una pieza textil interactiva, se procedió a trabajar con la agrupación de Artesanas de Melipilla. Para seguir el lineamiento del proyecto, era de vital importancia trabajar en conjunto con expertas artesanas y no "imitar" la técnica. Si se consideraba la artesanía no solo como un "saber hacer" pero como la materialización de un patrimonio cultural, el punto de vista y conocimiento de la agrupación era fundamental para entender de mejor manera el posible vínculo con el Diseño y nuevas tecnologías y sus posibles resultados. La etapa de diseño y fabricación de la pieza estuvieron guiadas por el concepto de co-creación. Tanto diseño como artesanía debían conversar y nutrirse de los conocimientos mutuos, donde ambas partes eran igualmente relevantes, con espacio para proponer y crear en conjunto. Para ello, se abrió un espacio de encuentro y conversación, en la cual la propuesta era debatida. Luego, se generó un traspaso de conocimientos técnicos desde cómo es la creación de una arpillera y cuáles son los materiales y puntadas hasta cómo utilizar el hilo conductor e integrar los componentes electrónicos a la tela. La creación finalmente se dividió en etapas de diseño, incorporación de componentes electrónicos, bordado, programación y terminaciones finales.

Durante todo el proceso, se resguardó el mantener un espacio de conversación transparente, para clarificar los objetivos de artesanas y diseñadora, y reconocer las distintas competencias pero en las mismas condiciones.

\section{Desarrollo}

A. Estudio materiales, componentes, procesos y electrónica básica La primera etapa tuvo como objetivo ahondar en el mundo de los textiles electrónicos, donde se profundizaron los conocimientos sobre componentes, 
Tabla 1. Softwares, materiales, herramientas y procesos de fabricación estudiados.

Fuente: elaboración propia

\begin{tabular}{|c|c|c|c|c|c|}
\hline Software & Materiales & Herramientas & Sensores & Actuadores & Reguladores \\
\hline $\begin{array}{l}\text { Arduino } \\
\text { IDE }\end{array}$ & $\begin{array}{l}\text { Tela } \\
\text { conductora }\end{array}$ & Multímetro & Fotorresistencia & LED & Resistencias \\
\hline \multirow[t]{6}{*}{ Fritzing } & Tela resistora & $\begin{array}{l}\text { Fuente de } \\
\text { poder }\end{array}$ & Botones & Neopixel & Diodos \\
\hline & Hilo conductor & Router CNC & Sensor presión & Reproductor $\mathrm{MP}_{3}$ & Transistores \\
\hline & Placas cobre & Cautín & Sensor tacto & Parlante & Baterias \\
\hline & Agujas & & Potenciómetro & Motor Vibrador & \\
\hline & $\begin{array}{l}\text { Pintura } \\
\text { aislante }\end{array}$ & & & & \\
\hline & Soldadura & & & & \\
\hline
\end{tabular}

materiales, procesos de fabricación y electrónica básica. Este proceso de estudio e investigación estuvo acompañado del desarrollo de muestras físicas en pequeños formatos. Durante esta etapa fue relevante hacer un levantamiento de información sobre los materiales disponibles en el mercado y las versiones listas o "Hazlo tú mismo" como, por ejemplo, el caso de un sensor de presión. Particularmente se decidió por experimentar con las versiones DIY (Hazlo tú mismo), lo que permitió mayor exploración y menores costos asociados.

\section{B. Vinculación con artesanas}

Para lograr un trabajo en conjunto con artesanas expertas, el proyecto fue desarrollado en conjunto con la agrupación Arpilleristas de Melipilla. Este grupo, liderado por María Cartes, cuenta actualmente con diez mujeres iniciadoras de la técnica con cerca de cuarenta años de experiencia. La posibilidad de poder sumar capas de información a sus relatos textiles, ya que crea un soporte que no solo se mira sino que además se toca, se escucha y se siente, fue la propuesta presentada por la diseñadora. Frente a ello, la respuesta fue positiva en cuanto ver el espacio como una oportunidad para explorar y tal vez acercar su arte a un nuevo público dándole, en sus propias palabras, "aún más vida a la arpillera". El proceso de trabajo en conjunto tuvo una etapa inicial de intercambio de conocimientos entre diseñadora y artesanas, se realizaron sesiones de bordado y muestras de incorporación de electrónica al textil. Estos encuentros fueron realizados en la sede de trabajo de la agrupación, en la ciudad de Melipilla.

\section{Creación de la pieza}

Con un entendimiento inicial de las técnicas por parte de artesanas y diseñadora, se planificó la creación de la pieza textil que siguió una lógica de colaboración. Las decisiones creativas y técnicas se tomaron en conjunto, como también la ejecución durante todo el proceso.

Se decidió crear una arpillera de gran formato de $1000 \mathrm{~cm} \times 70 \mathrm{~cm}$ que sería trabajada por etapas, las cuales se exponen a continuación:

\section{1) Diseño}

El primer paso es decidir la temática y narrativa de la pieza, la cual es llevada adelante por las artesanas quienes consideran que el público inicial que verá la pieza es internacional, por lo que deciden mostrar "la belleza de Chile". La historia que se relata entonces es la vida del campo con siete es- 

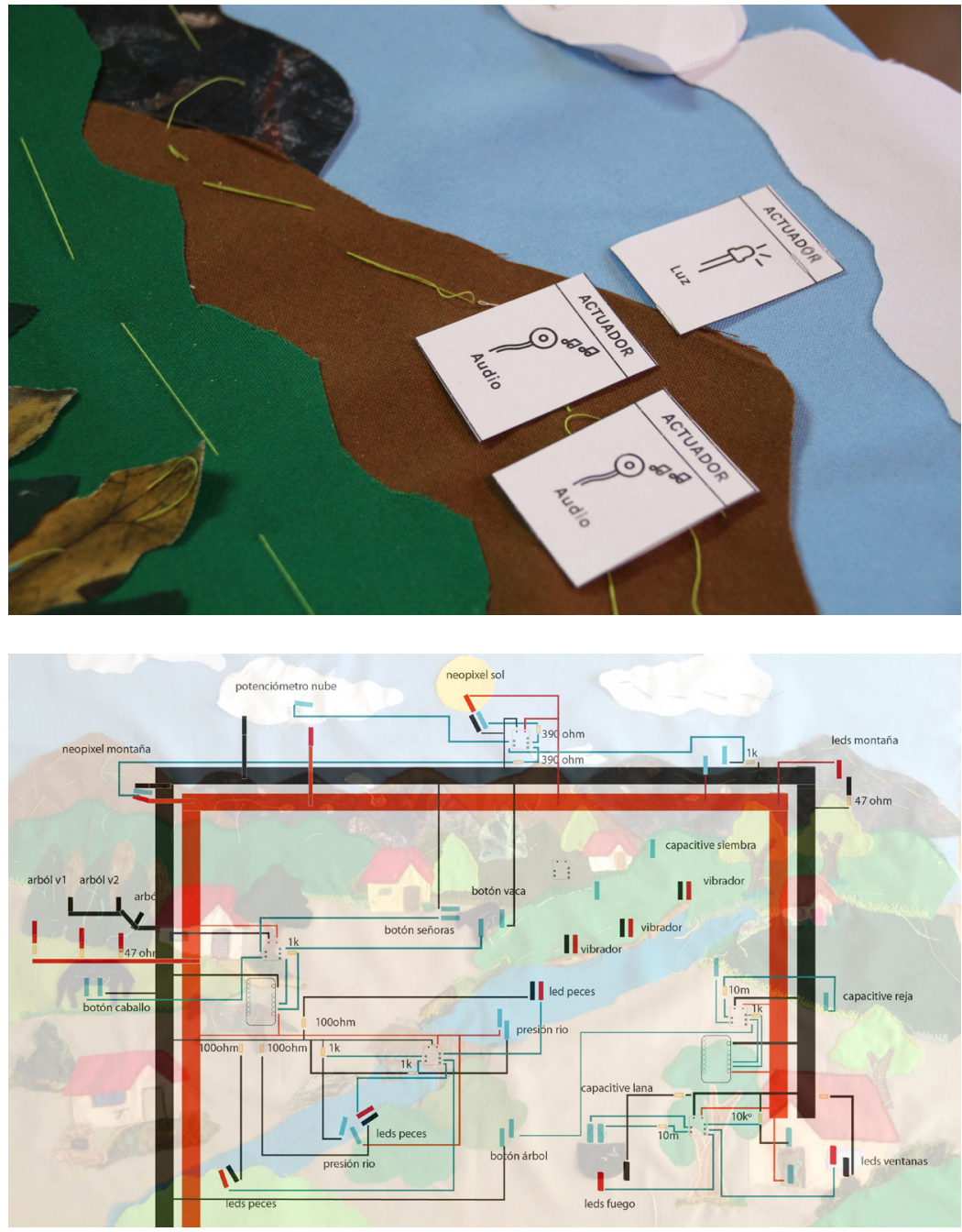

cenas contextualizadas en el valle central: fabricación del pan, fabricación de lana, plantaciones, criadero de ovejas, huaso y su caballo, y animales pastando. En este proceso, ya se comienza a pensar cuales serán las acciones relacionadas usando electrónica, como por ejemplo el brillo del sol o una conversación entre dos personajes.

Para la configuración de la obra, se sigue el esquema tradicional de cielo, cordillera y tierra, se crea una maqueta de textiles de figuras cortadas y posiciones sobre el fondo.

Otro tema importante a tratar en esta etapa es si mantener la electrónica escondida o a la vista. Junto a las artesanas, se decide "esconderla" de manera que sea una sorpresa para el espectador y no altere el lenguaje visual tradicional.

\section{2) Interacciones}

Para definir las interacciones basadas en la electrónica como, por ejemplo, al tocar a un personaje, suena un diálogo; se utilizan iconos especialmente diseñados, los cuales se posicionan sobre la maqueta de arpillera.
Figura 3. Interacciones. Fuente: Sofía Guridi. Figura 4. Creación circuito electrónico. Fuente: Sofía Guridi. 


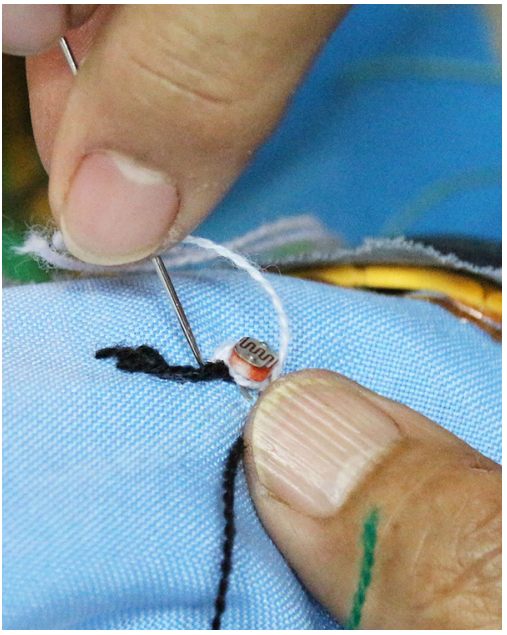

Figura 5. Bordado electrónica. Fuente: Sofía Guridi. Figura 6. Circuito exterior. Fuente: Sofía Guridi.

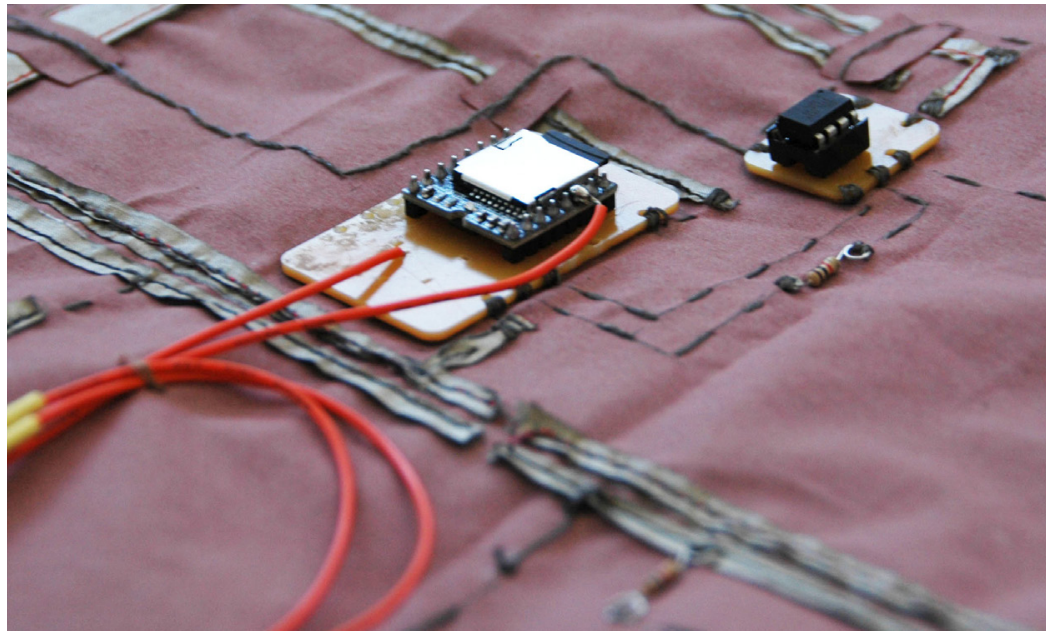

3) Creación de componentes y del esquemático

Si se considera la posición de los componentes, se diseña el esquemático eléctrico de conexiones y fabrican los componentes a utilizar.

\section{4) Bordado electrónico}

Sobre la base de textil, se integra la electrónica: sensores, actuadores, microcontroladores y reguladores de corriente. Para ello, se utilizan técnicas de bordado con hilo conductor, con cuidado de evitar posibles cortocircuitos. Algunos de estos componentes deben integrarse directamente en el bordado de lana partida, como es el caso del sensor de luz integrado en la cabeza de un cóndor. A medida que se posicionan los elementos, se testean con un computador, fuente de poder y microcontrolador Arduino.

Este proceso es llevado a cabo principalmente por la diseñadora, pero se dejan instancias de participación de artesanas, de manera que puedan al experimentar con el uso de hilo conductor y los componentes, entregar comentarios sobre la ejecución.

\section{5) Bordado textil}

Sobre la base textil, con la mayor parte de la electrónica incorporada, se pasa al bordado final de los recortes de figuras en tela utilizando las puntadas tradicionales de la arpillera.

Este proceso es iterativo y flexible, ya que es necesario ajustar algunas figuras, cantidad de capas de tela y posición de algunos componentes de la electrónica para que la unión de todas las partes genere un resultado correcto.

\section{6) Circuito exterior}

Una vez terminada la pieza textil con la electrónica incorporada, el último paso es unir todos los terminales de los componentes que fueron dejados hacia el exterior con tela e hilo conductor, para crear el circuito final unido a los microcontroladores y entradas de energía.

\section{7) Programación}

Con el circuito listo y los componentes conectados, se termina el trabajo con la programación final de los microcontroladores incorporados, donde se usa la plataforma Arduino IDE. 


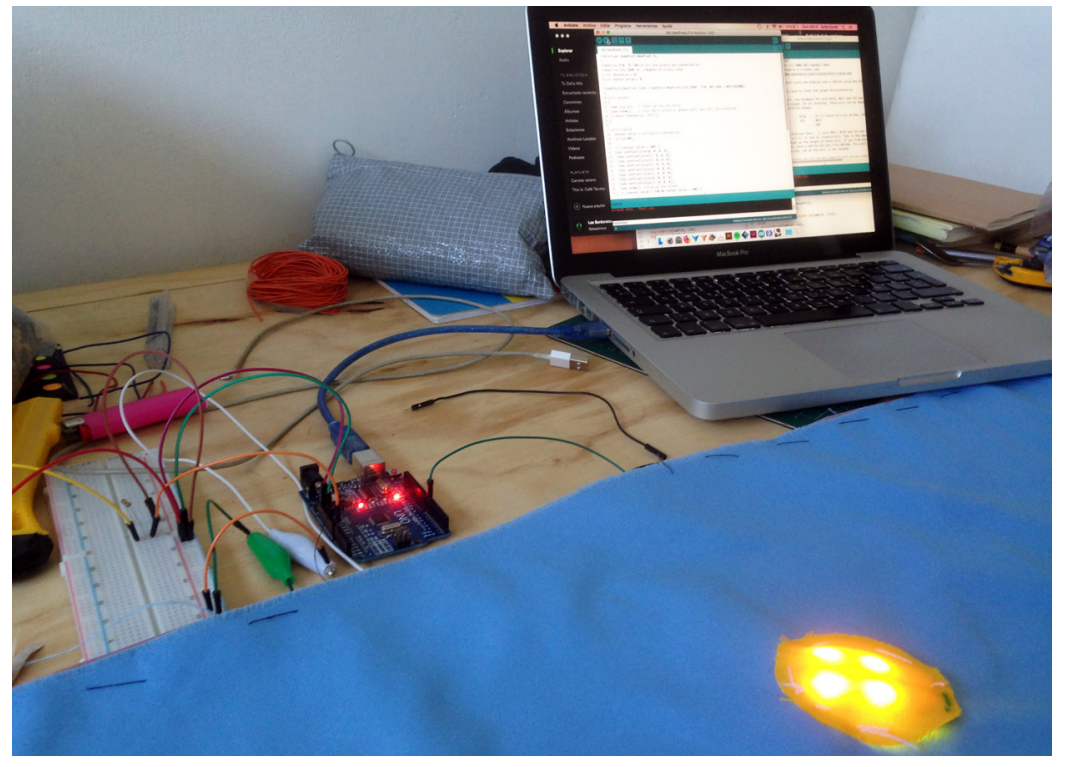

\section{ACCIÓN ESPECTADOR}

Acerca mano a águila en el cielo

Tocar contorno montaña

Tocar ovejas

Tocar caballo

Tocar personaje femenino

Tocar río

Abrir puerta casa

Tocar lana olla

Tocar caballo

Tocar árboles

\section{Resultados}

Luego de un proceso de creación conjunta de dos meses guiado por la diseñadora a cargo, el resultado es una arpillera interactiva de $1 \times 0,7 \mathrm{mts}$. En el siguiente diagrama se detallan las interacciones elegidas, las cuales determinaron qué componentes usar y su posición en el textil.

El funcionamiento de la arpillera está dado por la creación de circuitos eléctricos conectados a una única fuente de poder de $5 \mathrm{~V}$ (cargador convencional de celular). Debido a los componentes seleccionados, la pieza es capaz de responder a cambios de luz, tacto y a la presión. Estos sensores gatillan sonidos, movimiento o luz, lo que depende del caso.

El detalle de estas interacciones se observan en la tabla 2.

En la siguiente imagen se detallan las interacciones elegidas, las cuales determinaron qué componentes usar y su posición en el textil.

\section{Conclusiones}

Si se considera que se trata de un primer ejercicio de exploración, los resultados son positivos e inspiradores. Al seguir el objetivo principal, la obra textil resultante refleja que es posible la vinculación entre nuevas tecnologías de e-textiles y una técnica textil tradicional, tanto a nivel conceptual como
Figura 7. Programación. Fuente: Sofia Guridi.

Tabla 2. interacciones arpillera.

Fuente: elaboración propia. 
Figura 8. Arpillera final detalle. Fuente: Sofía Guridi. Figura 9. Arpillera final general. Fuente: Sofía Guridi. Figura 10. Exhibición Museo Violeta Parra 2019. Fuente: Pablo Fuente.
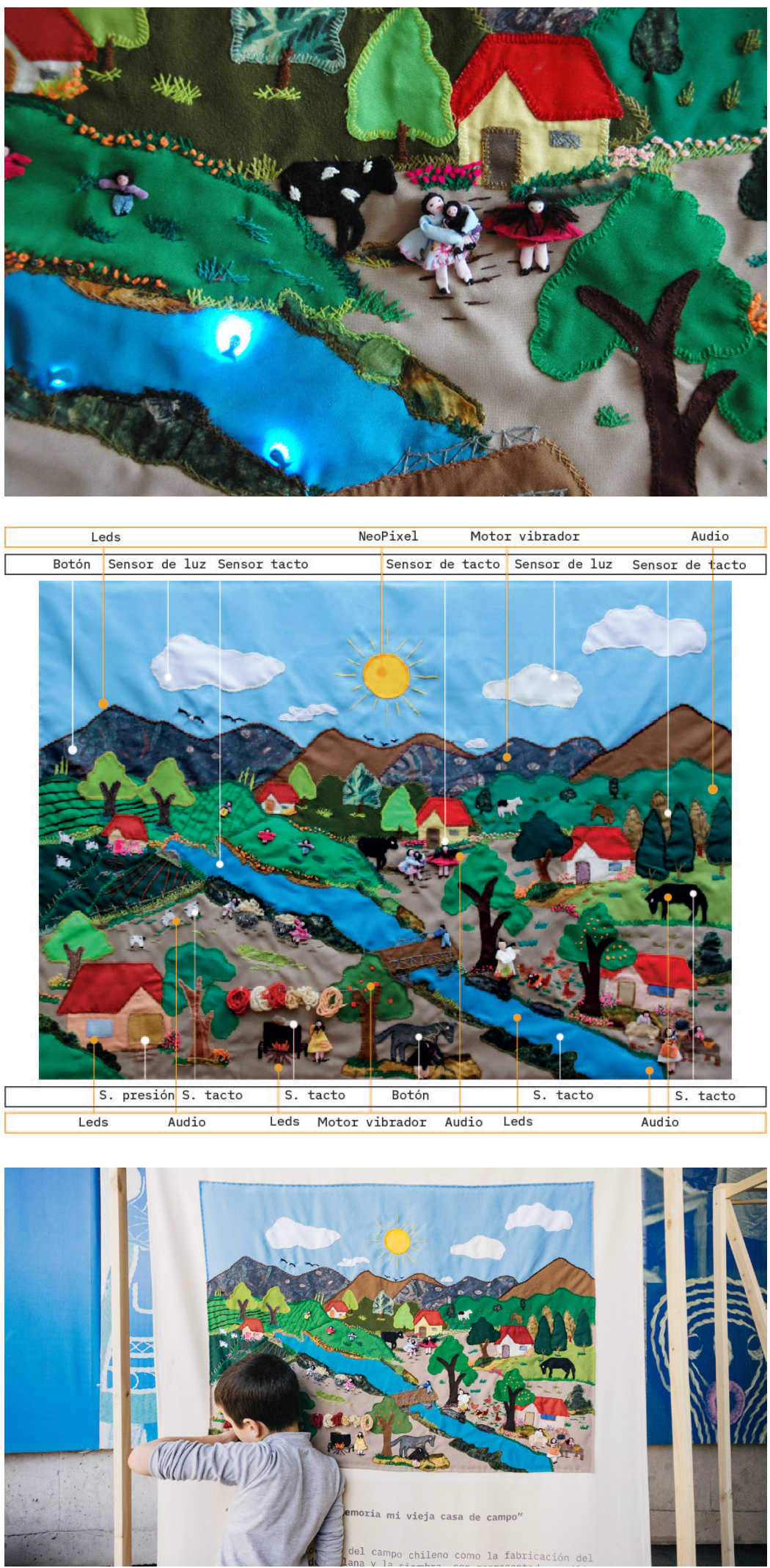
técnico. Gracias al trabajo en conjunto con artesanas fue posible crear una pieza textil interactiva, para potenciar el carácter narrativo e identitario de la información visual, con información táctil y auditiva, que revela mensajes "secretos" al estar en contacto con el espectador. El vínculo entre el observador y la obra se fortalece al permitir al primero descubrir nuevos mensajes y generar respuestas según su movimiento corporal, los cuales no son esperados. Este factor de sorpresa se debe a que el aspecto principal de la pieza no varía desde su concepción tradicional. Si bien integra nuevos elementos en segundo plano, los códigos visuales, materialidades y técnica se mantienen. En términos técnicos, el resultado es positivo. La electrónica logra ser integrada y es interesante ver cómo los nuevos materiales programados a nivel digital sustentan la experiencia táctil. El cuerpo está presente en el proceso de fabricación, a través del bordado de hilos conductores o de placas electrónicas en el cual las manos todavía son fundamentales, lo que permite así el conocimiento a través del cuerpo, lo cual es clave en la artesanía y el diseño. En este caso, es principalmente la electrónica la que se modifica para integrarse al bordado y textil, por lo cual sería interesante en otras instancias, experimentar aún más para ver si finalmente ambas técnicas permean entre sí, para generar como resultado una arpillera con un lenguaje visual transformado. Se debería reflexionar cómo podría impactar esto en la artesanía, y evaluar el trabajo de esta perspectiva en sintonía con artesanas. Los problemas técnicos se debieron principalmente a la construcción por capas. Por la forma en que fue planeada, es complejo acceder a la electrónica en caso de haber elementos que no estén funcionando de manera correcta. Por otro lado, se debe optimizar la utilización de tela conductora en la construcción del circuito de alimentación de corriente principal, ya que la resistencia de la misma es muy alta, lo que ocasiona algunos problemas de funcionamiento. Respecto al trabajo entre diseñadora y artesanas, fue posible generar una instancia de aprendizaje mutuo, lo que enriquece la creación en conjunto de una pieza en la que ambas partes son creadoras por igual. Sus comentarios finales mostraron asombro por lo logrado, ya que al no haber referentes era bastante difícil imaginar el resultado final. Queda por resolver, sin embargo, cómo entregar las herramientas necesarias para que las artesanas puedan seguir desarrollando estos nuevos soportes. Se consideró el uso de componentes de audio fáciles de manejar, para agregar en las tarjetas, pero no fue posible desarrollar en profundidad este aspecto.

Por último, a partir de las reacciones finales de las artesanas, se desprende la necesidad de generar instancias de encuentro orientadas a apoyar la transmisión de su conocimiento a nuevos públicos. Una de sus principales motivaciones para participar de este proyecto fue el deseo de tener espacios de encuentro y personas interesadas con quienes compartir su pasión por la arpillera, en particular con grupos más jóvenes. Sumado a esta inquietud y a la potencialidad de acercar la electrónica y programación a más personas desde una mirada cercana gracias al textil, es que se inicia el proyecto PIN "Arpilleras Parlantes" con financiamiento Fondart Nacional, Convocatoria 2019. A la fecha, los resultados del proyecto expuesto y proyecto Fondart (con foco en educación), han sido exhibidos en Fabricademy, Museo Violeta Parra, Fab Lab U. de Chile y II Encuentro Internacional de Arte Textil y Resistencia, que tuvo una recepción positiva, para así abrir nuevas posibilidades de trabajo en espacios nacionales como internacionales para seguir explorando y mostrar a un público más amplio el trabajo de artesanas chilenas desde otra perspectiva. 
Finalmente, se aprecia cómo esta experiencia abre un nuevo camino de exploración con potencial para el enriquecimiento de la arpillera en la unión con nuevas tecnologías interactivas. Sin embargo, será necesario continuar con nuevas experiencias de creación y evaluar desde un visión crítica y con tiempo cuál sería el real impacto de este desarrollo en la comunidad de artesanas, en la artesanía en sí misma y en el desarrollo de nuevas tecnologías de e-textiles. Para ello, será relevante, además, profundizar en la metodología de trabajo en conjunto, ahondando en la identidad y proceso creativo de los involucrados.

\section{Agradecimientos:}

Cecilia Raspanti

Anastasia Pistofidou

Adriana Cabrera

Arpilleristas de Melipilla

Fab Lab Santiago

\section{References}

Agosín, M. (1985). “Agujas que hablan: las arpilleristas chilenas". Revista Iberoamericana Vol. LI. pp. 132-133.

Berzowska, J. (2005). “Electronic Textiles: Wearable Computers, Reactive Fashion, and Soft Computation". Textiles: The Journal of Cloth and Culture 3(1) pp. 58-75.

Museo de la Memoria y los Derechos Humanos. (2012). Arpilleras. Santiago: Ocho Libros Editores.

Nimkulrat, N. (2012). "Hands-on Intellect: Integrating Craft Practice into Design Research". International Journal of Design 6(3) pp. 1-14.

Organización de las Naciones Unidas para la Educación, la Ciencia y la Cultura (UNESCO). (2009). Artesanía y Diseño: Taller $A+D\left(N^{\circ} 2\right)$. Recuperado de http://www.unesco.org

Consejo Nacional de la Cultura y de las Artes (CNCA). (2017). Política de Fomento del Diseño 2017-2022. Santiago: CNCA.

Ghahremani M. \& Latifi M. (2016). "Overview of wearable electronics and smart textiles". The Journal of The Textile Institute 108(4) pp. 1-22.

Guler, SD., Gannon, M., Sicchio, K. (2016). Crafting Wearables. USA: Apress Media.

Persson, A. (2013). Exploring textiles as materials for interaction design (Tesis de doctorado). University of Borâs, Borâs.

Schneegass \& S., Amft, O. (2017). Smart Textiles: Fundamentals, Design and Interaction. Springer International Publishing AC. Tao, X. (2001). Smart fibres, fabrics and clothing. Inglaterra: Woodhead Publishing Ltd and CRC Press LLC. 